\title{
RADÓ'S THEOREM FOR LOCALLY SOLVABLE VECTOR FIELDS
}

\author{
JORGE HOUNIE AND JOAQUIM TAVARES
}

(Communicated by Barbara L. Keyfitz)

Dedicated to $F$. Treves on the occasion of his sixtieth birthday

\begin{abstract}
We extend the classical theorem of Rado, valid for the CauchyRiemann operator, to locally solvable first-order operators with smooth coeffcients in $\mathbb{R}^{N}$.
\end{abstract}

\section{INTRODUCTION}

A classical theorem of Rado, in the form given by Cartan, states that a continuous function defined in an open set of the complex plane which is holomorphic outside the closed set where it vanishes is holomorphic everywhere. This theorem implies easily that the same result also holds for functions of several complex variables. Recently, Rosay and Stout [RS] obtained an analogue of Radó's theorem for certain classes of CR-functions of hypersurface type and formulated a general problem about vector fields which we now describe. Let $\Omega \subset \mathbb{R}^{N}$ be an open set and consider a vector field $L$ in $\Omega$. A continuous function $u \in C(\Omega)$ that satisfies the equation $L u=0$ in the sense of distributions on the set $\Omega \backslash u^{-1}(0)$ is called a Radó function. The vector fields $L$ for which all Radó functions are weak solutions of $L u=0$ throughout $\Omega$ are said to have the Radó property. For instance, the Cauchy-Riemann operator has the Radó property by the classical theorem of Radó. A similar definition can be given for systems of vector fields.

In this paper we prove that locally solvable vector fields with smooth coefficients in $\mathbb{R}^{N}$ have the Radó property. They are known to be characterized by the Nirenberg-Treves condition $(\mathscr{P})$ introduced in [NT]. The proof exploits two properties of this class of vector fields: its essentially two-dimensional character (expressed by the fact that orbits have dimension at most two) and the property of local integrability that allows reduction of the problem to the classical version of Radós theorem in the plane. However, we do not know if a general locally integrable vector field in two variables has the Radó property.

The paper concludes with an application to uniqueness in the Cauchy problem with irregular initial surface.

Received by the editors July 5, 1991 and, in revised form, October 17, 1991 and March 9, 1992. 1991 Mathematics Subject Classification. Primary 35F05, 35D99.

The first author was supported in part by $\mathrm{CNP}_{\mathbf{q}}$. 


\section{BACKGROUND ON SOLVABLE VECTOR FIELDS}

We deal with a complex smooth vector field $L$ defined on an open subset $\Omega$ of $\mathbb{R}^{n+1}, n \geq 1$. We assume that $L$ never vanishes in $\Omega$ and satisfies the Nirenberg-Treves condition $(\mathscr{P})$ [6], which we now describe. The principal symbol $l$ of $L$ is defined on the cotangent bundle $T^{*}(\Omega)$ by the identity $l(d \phi)=L(\phi), \phi \in C^{\infty}(\Omega ; \mathbb{R})$. Then $L$ is said to verify $(\mathscr{P})$ in $\Omega$ if there is no complex-valued function $g \in C^{\infty}(\Omega)$ such that $\operatorname{Im}(g l)$ takes both positive and negative values on a null bicharacteristic of $\operatorname{Re}(g l)$ where $g \neq 0$. We recall that a bicharacteristic of a real function $f$ defined on $T^{*}(\Omega)$ is an integral curve of the Hamiltonian field $H_{f}$. Since $H_{f} f=0, f$ is constant along its bicharacteristics; when the constant is zero the bicharacteristic is said to be null.

Let us write $X=\operatorname{Re} L, Y=\operatorname{Im} L$. The orbits of the pair of vector fields $X$ and $Y$ in the sense of Sussmann [S] are called the orbits of $L$. Two points belong to the same orbit if and only if they can be joined by a continuous piecewise differentiable curve such that each piece is an integral curve of $\pm X$ or $\pm Y$. The orbits are connected submanifolds of $\Omega$, tangent to $X$ and $Y$, and can be used to characterize the vector fields that satisfy $(\mathscr{P})[$ Ho, Theorem 3.1]. In particular, if $L$ satisfies $(\mathscr{P})$, the orbits of $L$ are submanifolds of dimension 1 or 2 . If $u$ is a distribution solution of $L u=0$ in $\Omega$, the support of $u$ is a union of orbits of $L$. This follows from the fact that if $L$ satisfies $(\mathscr{P})$, it has uniqueness in the Cauchy problem with respect to noncharacteristic surfaces in the class of distributions [CH] (actually, we will only be interested in continuous weak solutions). In fact, this uniqueness property holds for the more general class of locally integrable vector fields $[\mathrm{T}]$. We recall that $L$ is locally integrable if every point $p \in \Omega$ possesses a neighborhood $U$ such that there exist smooth functions $Z_{1}, \ldots, Z_{n} \in \Omega$ with linearly independent differentials satisfying $L Z_{j}=0, j=1, \ldots, n$. If $u \in C^{0}(U)$ then every point $q \in \Omega$ has a neighborhood $V$ where $u$ can be uniformly approximated by a sequence of polynomials $P_{j}(Z)=P_{j}\left(Z_{1}, \ldots, Z_{n}\right)$. This is an instance of the BaouendiTreves approximation theorem [BT1, BT2]. If $F$ is a fiber of $Z$ in $V$, i.e., a set of the form $Z^{-1}(\zeta) \cap V$ with $\zeta \in Z(V)$, it follows that $u$ is constant of $F$.

Definition 1.1. Given a vector field $L$ in $\Omega \subset \mathbb{R}^{N}$ we say that $u \in C(\Omega)$ is a Radó function for $L$ if

$$
L u=0 \quad \text { on } \Omega \backslash u^{-1}(0)
$$

in the sense of distributions. If every Radó function for $L$ is a weak solution of $L u=0$ in $\Omega$, we say that $L$ has the Radó property.

Notice that the property of Radó is of local nature.

Lemma 1.2. Let $L$ be locally integrable, and let $u$ be a Radó function for $L$. Then $u$ is constant on the orbits of $L$ of dimension one.

Proof. Let $\gamma$ be an orbit of $L$ of dimension one. If $u$ vanishes identically in $\gamma$, there is nothing to prove. Consider a point $p \in \gamma$ such that $u(p) \neq 0$, and choose local coordinates $(x, t)$ in a neighborhood of $p$ such that $x(p)=$ $t(p)=0$ and $Z_{j}(x, t)=x_{j}+i \phi_{j}(x, t), j=1, \ldots, n,|x|<1,|t|<1$. After 
multiplying $L$ by a convenient nonvanishing function we may assume that $L=\partial_{t}+\sum_{1}^{n} \lambda_{j} \partial_{x_{j}}$ where the coefficients $\lambda_{j}$ are determined by the equations $L Z_{j}=0, j=1, \ldots, n$. Since $X$ and $Y$ are linearly dependent on $\gamma$ and this is only possible where $\lambda_{j}=0, j=1, \ldots, n$, we conclude that in our coordinate patch $\gamma$ can be parametrized by $\{(0, s):-1<s<1\}$ (note that the integral curve of $X$ through the origin is contained in $\gamma)$. Hence, the equations $L Z_{j}=0$ when restricted to $\gamma$ mean that $\partial_{s} Z_{j}=0$, which implies that $\gamma$ is contained on a fiber of $Z$. Since $u$ is a Radó function and $u(0) \neq 0$, we know that $L u=0$ for $|x|,|t|<\varepsilon$ for a certain $\varepsilon>0$ and, by the remarks made above, that $u$ must be constant in $\gamma$ for $|s|$ small. By connectedness it follows that $u$ is constant throughout $\gamma$.

We will also make use of the following observation. Let $P\left(x, y, D_{x}\right)$ be a differential operator with coefficients depending on $x \in \mathbb{R}^{n}$ and $y \in \mathbb{R}^{m}$ containing derivatives just with respect to $x$. If $u(x, y)$ is a continuous weak solution of $P\left(x, y, D_{x}\right) u=0$ in $\mathbb{R}^{n+m}$, then $v(x)=u\left(x, y_{0}\right)$ is a weak solution of $P\left(x, y_{0}, D_{x}\right) v=0$ in $\mathbb{R}^{n}$. For the proof it is enough to consider test functions of the form $\phi(x) \psi(y)$, observe that $P^{\mathrm{t}}(\phi \psi)=\psi P^{\mathrm{t}}(\phi)$, and then use Fubini's theorem and a density argument to conclude that $\int v(x) P^{\mathrm{t}}(\phi) d x=0$ $\left(P^{\mathrm{t}}\right.$ is the transpose of $P$ ). In particular, assume that $L$ is a vector field defined on an open set $U \subset \mathbb{R}^{n}$ and that $L$ is tangent to the leaves of a regular foliation of $U$. If a continuous function $u$ satisfies $L u=0$ in $U$ in the sense of distributions and $\Sigma$ is a leaf of the foliation, then the restriction $v$ of $u$ to $\Sigma$ satisfies $L v=0$ in $\Sigma$ (we are identifying $L$ and its pullback to $\Sigma$ ).

\section{ThE MAIN RESUlT}

In this section we prove

Theorem 2.1. Let $L$ be a vector field with smooth coefficients in $\Omega \subset \mathbb{R}^{n+1}$ satisfying condition $(\mathscr{P})$. Then $L$ has the property of Radó.

After localization, choice of appropriate coordinates, and multiplication of $L$ by a nonvanishing factor we may write

$$
L=\frac{\partial}{\partial t}-i \sum_{j=1}^{n} b_{j}(x, t) \frac{\partial}{\partial x_{j}}, \quad|x| \leq 1,|t| \leq 1,
$$

where the coefficients $b_{j}(x, t)$ are real smooth functions. We denote by $\vec{b}(x, t)$ the vector field in $\mathbb{R}^{n}$ given by $\sum_{j=1}^{n} b_{j}(x, t) \partial / \partial x_{j},|x|,|t| \leq 1$. The fact that $L$ verifies $(\mathscr{P})$ implies that there exists a unit vector field $\vec{v}(x)$ defined on $\mathbb{R}^{n}$ for $|x| \leq 1$ such that

$$
\vec{b}(x, t)=|\vec{b}(x, t)| \vec{v}(x), \quad x \in \mathbb{R}^{n}, t \in \mathbb{R},|x|,|t| \leq 1 .
$$

Set

and

$$
\mathscr{N}=\left\{x \in \mathbb{R}^{n}: \vec{b}(x, t)=0,|x|,|t|<1\right\}
$$

$$
\rho(x)=\sup _{|t|<1}|\vec{b}(x, t)|, \quad x \in \mathbb{R}^{n},|x|<1,
$$

so that $\mathscr{N}$ is precisely the set where $\rho$ vanishes. The function $\rho(x)$ is Lipschitz and $\|\nabla \rho\|_{L^{\infty}} \leq\left\|\nabla_{x} \vec{b}\right\|_{L^{\infty}}$. Assuming, without loss of generality, that 
$\|\nabla \vec{b}\|_{L^{\infty}} \leq 1$ we obtain the following estimate that we note for later reference:

$$
\left|\rho(x)-\rho\left(x^{\prime}\right)\right| \leq\left|x-x^{\prime}\right|, \quad x, x^{\prime} \in \mathbb{R}^{n},|x|,\left|x^{\prime}\right|<1 .
$$

In the next lemma $L^{\mathrm{t}}$ indicates the transpose of $L$.

Lemma 2.2. Let $\chi$ be the characteristic function of $\mathscr{N}$, and let $\phi \in C_{c}^{\infty}\left(\mathbb{R}^{n+1}\right)$ be supported in the unit cube $Q$. Assume that the continuous function $u$ satisfies $L u=0$ where $u \neq 0$. Then

$$
\iint u(x, t) \chi(x) L^{\mathrm{t}} \phi d x d t=0 .
$$

Proof. If $x \in \mathscr{N}$, the segment $\{x\} \times(-1,1)$ is contained in an orbit of $L$ of dimension one. By Lemma 1.2 we conclude that the restriction of $u$ to $\mathscr{N} \times(-1,1)$ is a function of $x$ alone. Furthermore, $L^{\mathrm{t}}=-\partial / \partial t+c(x, t)$ if $x \in \mathscr{N}$, where $c(x, t)=i\left(\sum_{k} \partial b_{k} / \partial x_{k}\right)$. We may write $\mathscr{N}=\mathscr{N}_{1} \cup \mathscr{N}_{2}$ with

$$
\mathscr{N}_{1}=\left\{x \in \mathscr{N}: \exists t, \nabla_{x} \vec{b}(x, t) \neq 0\right\}
$$

and $\mathscr{N}_{2}=\mathscr{N} \backslash \mathscr{N}_{1}$. By the implicit function theorem $N_{1}$ is a negligible set and $L^{\mathrm{t}} \phi=-\phi_{t}$ on $\mathscr{N}_{2} \times(-1,1)$. If we let $x$ vary over $\mathscr{N}_{2}$ in $(2.3)$ and integrate first in $t$ we obtain (2.3).

Our next step is to prove that

$$
\iint u(x, t)(1-\chi(x)) L^{\mathrm{t}} \phi d x d t=0, \quad \phi \in C_{c}^{\infty}(Q) .
$$

Let $Q_{1}$ be the open cube $|x|<1$ in $\mathbb{R}^{n}$. Consider a sequence of functions $\phi_{k}(x) \in C_{c}^{\infty}\left(Q_{1} \backslash \mathscr{N}\right)$ such that $\phi_{k}(x)=1$ if $\operatorname{dist}(x, \mathscr{N})>1 / k$ and $\left|\nabla \phi_{k}(x)\right| \leq$ $C k$ where $C>0$ is independent of $k$. This sequence is easily constructed.

Lemma 2.3. For $k=1,2, \ldots$

$$
\iint u(x, t) L^{\mathfrak{t}}\left(\phi_{k} \phi\right) d x d t=0, \quad \phi \in C_{c}^{\infty}(Q) .
$$

We postpone the proof of Lemma 2.3 to the next section and continue our reasoning. Letting $k \rightarrow \infty$ in (2.5) we observe that the integrand converges pointwisely and boundedly to $u(x, t)(1-\chi(x)) L^{\mathfrak{t}} \phi$. This is so because the derivatives with respect to $x_{j}$ of $\phi_{k}(x)$, which are bounded by $C k$, appear as factors of the coefficients $b_{j}$, which are majorized by $\rho(x)$, and since the support of $\nabla \phi_{k}$ is contained in a $1 / k$-neighborhood of $\mathscr{N}$, it follows from (2.2) that $\rho \leq 1 / k$ on the support $\nabla \phi_{k}$. Hence, the dominate convergence theorem applies and (2.5) implies (2.4) when $k \rightarrow \infty$. Finally, adding (2.3) and (2.4) we obtain that $L u=0$ in the sense of distributions in $Q$.

\section{Proof of Lemma 2.3}

Since $\phi_{k} \phi \in C_{c}^{\infty}(Q \backslash \mathscr{N} \times(-1,1)),(2.5)$ will follow if we prove that $u$ is a weak solution of $L u=0$ in $\Omega \backslash \mathscr{N} \times(-1,1)$ under the assumption that $u$ is a Radó function. Every point of $Q \backslash \mathscr{N} \times(-1,1)$ is contained in a two-dimensional orbit of $L$ in $Q$ because all one-dimensional orbits are contained in $\mathscr{N} \times(-1,1)$. In a neighborhood of such a point we may choose the coordinates so that the two-dimensional orbits are given by $x_{j}=$ constant , 
$j=2, \ldots, n$. This is easily achieved by (locally) rectifying the flow of $\vec{v}$, which is smooth outside $\mathscr{N}$. In such coordinates, $L$ assumes the form

$$
\frac{\partial}{\partial t}-i b(x, t) \frac{\partial}{\partial x_{1}}, \quad|x| \leq a,|t|<1,
$$

with $b(x, t) \geq 0$. Furthermore,

$$
\begin{aligned}
& \text { For all } x \in[-a, a] \text { the function }(-1,1) \ni t \rightarrow \\
& b(x, t) \text { does not vanish identically. }
\end{aligned}
$$

Considering $x_{2}, \ldots, x_{n}$ as parameters we may now deal with a vector field in two dimensions (see the observation at the end of $\S 1$ ). To simplify the notation we write $x$ instead of $x_{1}$ and consider a vector field

$$
L=\frac{\partial}{\partial t}=i b(x, t) \frac{\partial}{\partial x}, \quad(x, t) \in \mathbb{R}^{2},
$$

with $b(x, t) \geq 0$ defined in a neighborhood of $\bar{\Omega}=[-a, a] \times[-T, T]$. Solving the equation $L U=i b_{x}$ in a neighborhood of $\bar{\Omega}$ we may construct a smooth function $Z$ such that

$$
L Z=0, \quad Z_{x} \neq 0, \quad(x, t) \in \Omega .
$$

This is the property of locally integrability. We also assume that (3.1) holds. The construction and basic properties of $Z$ are thoroughly explained in $[\mathrm{H}$, Corollary 4.5] and the following discussion (cf. also [C, p. 12]). We wish to prove that $L u=0$ in the sense of distributions. Since this is a local property, it may be studied in the neighborhood of a given point $p \in \Omega$. If $L$ is elliptic at $p \in \Omega$ then $L$ is a multiple, in convenient new local coordinates, of the CauchyRiemann operator. By the classical theorem of Radó $L u=0$ in a neighborhood of $p$. From now on we assume that $L$ is not elliptic at $p$, which we take to be the origin without loss of generality. Thus, assume that $b(0,0)=0$ and let $\left[t_{0}, t_{1}\right]$ be the maximal closed subinterval of $[-T, T]$ containing the origin on which $b(0, t)$ vanishes. Observe that $\left[t_{0}, t_{1}\right]$ may reduce to $\{0\}$ but cannot be equal to the whole $[-T, T]$ in view of $(3.1)$, say $t_{1}<T$. On $I=\{0\} \times\left[t_{0}, t_{1}\right]$ the vector field $L$ reduces to $\partial / \partial t$ and $Z_{x}$ is constant. To check this observe that since $b(0, t)$ vanishes on $I=\{0\} \times\left[t_{0}, t_{1}\right], t_{0}<0<t_{1}$, and $b \geq 0$, it follows that also $b_{x}$ vanishes on $I=\{0\} \times\left[t_{0}, t_{1}\right]$. Differentiating the equation $Z_{t}-i b Z_{x}=0$ with respect to $x$ and setting $x=0$ we obtain $\partial_{t}\left(Z_{x}(0, t)=0\right.$ for $t \in\left[t_{0}, t_{1}\right]$, which implies that $Z_{x}$ is constant on $I$.

Using $(\operatorname{Re} Z, t)$ as new coordinates in a neighborhood of $I$ we may assume, after redefining $a$ and $T$, that we are in the following situation:

(i) $Z(x, t)=x+i \phi(x, t)$ with $\phi(x, t)$ real valued, $|x| \leq a,|t| \leq T$;

(ii) $\left|\phi_{x}(x, t)\right|<\frac{1}{2},|x| \leq a,|t| \leq T$;

(iii) the function $t \rightarrow \phi(x, t)$ is monotone nondecreasing and not constant for any $x$; and

(iv) $L=\partial / \partial t-B(x, t) \partial / \partial x$ with $B(x, t)=i \phi_{t} /\left(1+i \phi_{x}\right)$.

Let us see why property (ii) is relevant for us. Let $R$ be a rectangle, with sides parallel to the coordinate axis, contained in $|x| \leq a,|t| \leq T$, and assume that $u$ satisfies $L u=0$ in a neighborhood of $R$. Then the Baouendi-Treves formula gives a sequence of entire functions of one complex variable $v_{k}(\zeta)$ such 
that $v_{k} \circ Z$ converges uniformly to $u$ on $R$ provided that $\left|\phi_{x}\right|<\frac{1}{2}$. That this can be achieved is easily seen by recalling that we may, in the first place, choose $Z$ so that $\phi_{x}(0,0)=0$, and this implies that $\phi_{x}$ vanishes throughout $I$. Then $\phi_{x}$ will have the required property in a neighborhood of $I$. Concerning (iii) see $[\mathrm{H}]$.

Lemma 3.1. The function $u$ is constant on the fibers of $Z$. Hence we may write $u=v \circ Z$ with a uniquely determined $v \in C^{0}(Z(\bar{\Omega}))$.

Proof. Notice that because of (iii) the fibers of $Z$ are of the form $F=\{x\} \times$ $[b, c]$ with $t \rightarrow \phi(x, t)$ constant in $[b, c]$. In particular, $L=\partial / \partial t$ on $F$. Then, on the open set $(-a, a) \times(b, c)$, the segment $\{x\} \times(b, c)$ is a onedimensional orbit of $L$ and Lemma 1.2 shows that $u$ must be constant on $\{x\} \times(b, c)$ and also on its closure $F$.

Lemma 3.2. The function $v$ of Lemma 3.1 is holomorphic on the open set

$$
U=\{\zeta=x+i y:-a<x<a, \phi(x,-T)<y<\phi(x, T)\} .
$$

Proof. By the classical version of Radó's theorem we need only show that $v$ is holomorphic where $v$ does not vanish. Assume

$$
\zeta=x+i \phi(x, t) \in U \text { and } v(\zeta)=u(x, t)=\lambda \neq 0 .
$$

If $\phi_{t}(x, t) \neq 0$ then $Z$ is a local diffeomorphism in a neighborhood of $(x, t)$ that takes $L$ into a multiple of $\partial / \partial \bar{\zeta}$ so $\partial v / \partial \bar{\zeta}=0$ in a neighborhood of $\zeta$. Suppose now that $\phi_{t}(x, t)=0$, and consider the fiber $F$ of $Z$ containing $(x, t)$. Then $u=\lambda$ on $F$ and $u \neq 0$ in a neighborhood of $F$. Choose a closed rectangle $R$ containing $F$ in its interior such that the equation $L u=0$ holds in a neighborhood of $R$ (the fact that $\zeta \in U$ implies that $F$ does not touch $\partial \Omega$ ). It is a consequence of the approximation theorem of Baouendi-Treves that $u=\tilde{v} \circ Z$ on $R$, with $\tilde{v}$ holomorphic in the interior of $Z(R)$. Now $Z(R)$ contains $\zeta$ in its interior and, of course, $\tilde{v}$ is just the restriction of $v$ to $Z(R)$. This proves the lemma.

Lemma 3.3. $L u=0$ in the sense of distributions in $\Omega$.

Proof. By Lemmas 3.1 and $3.2 u=v \circ Z$ with $v$ continuous in $\bar{U}$ and holomorphic in $U$. Using Mergelyan's theorem we may consider a sequence of polynomials $p_{k}(\zeta)$ converging uniformly to $v$ on $\bar{U}$. Then the functions $u_{k}=p_{k} \circ Z$ are smooth solutions of $L u_{k}=0$ on $\Omega$ that converge uniformly to $u$. This proves Lemma 3.3, which, as noted before, implies Lemma 2.3.

\section{UniQueness in the CAUChy PRoblem}

Let $L=X+i Y$ be a smooth complex vector field in $\Omega \subset \mathbb{R}^{n+1}$, let $U \subset \Omega$ be open, and denote by $\partial U$ the relative boundary of $U$ in $\Omega$.

Definition 4.1. We say that $\partial U$ is weakly noncharacteristic for $L$ if for every point $p \in \partial U$ the orbit of $L$ passing through $p$ intersects $\Omega \backslash \bar{U}$.

Note that if $\partial U$ is weakly noncharacteristic, $U$ is not dense in $\Omega$. If $\partial U$ is a regular (say $C^{1}$ ) noncharacteristic surface, at any point of $\partial U$ either $X$ 
or $Y$ is transversal to $\partial U$ and it is clear that $\partial U$ is weakly noncharacteristic. On the other hand, if $\partial U$ is regular and totally characteristic, i.e., $X$ and $Y$ are tangent to $\partial U$ everywhere, $\partial U$ is not weakly noncharacteristic.

Theorem 4.1. Let $L$ be a smooth complex vector field in $\Omega \subset \mathbb{R}^{n+1}$, and let $U \subset \Omega$ be open. Assume that $L$ satisfies $(\mathscr{P})$ and that $\partial U$ is weakly noncharacteristic. If $u$ is continuous in $U \cup \partial U$, satisfies $L u=0$ in $U$ in the sense of distributions and vanishes on $\partial U$, then $u$ vanishes in a neighborhood of $\partial U$.

Proof. Define $w \in C(\Omega)$ by $w=u$ in $U, w=0$ in $\Omega \backslash U$. Then $w$ is a Radó function for $L$ and by Theorem 2.1 it satisfies $L w=0$ in $\Omega$. Thus, the support $S$ of $w$ is a union of orbits of $L$ (see $\S 1$ ) and so is $\Omega \backslash S \supset \Omega \backslash \bar{U} \neq$ $\varnothing$. But the union of orbits that intersect $\Omega \backslash \bar{U}$ covers $\partial U$ because $\partial U$ is weakly noncharacteristic. Thus, $\Omega \backslash S$ is an open neighborhood of $\partial U$ where $u$ vanishes.

Remarks. (i) Theorem 4.1 weakens the hypothesis of previous theorems on uniqueness in the Cauchy problem for operators satisfying $(\mathscr{P})$, at least for continuous solutions, in the following way. First, no regularity is assumed on the initial "surface"; secondly, some regular points of the initial surface are allowed to be characteristic; and, finally, the function $u$ is assumed to verify the equation only on one side of the initial surface.

(ii) Theorems 2.1 and 4.1 are still valid for a general first-order operator with smooth coefficients. Indeed, if $L$ is a vector field satisfying $(\mathscr{P})$ and we consider the operator $P=L+c(x, t)$, the zeroth-order term can be absorbed because we can solve locally the equation $L v=c$ with, say, $v$ continuous, and then $L\left(e^{v} u\right)=0$ is equivalent to $P u=0$.

\section{ACKNOWLEDGMENT}

The authors wish to thank Professor F. Treves for several fruitful conversations on this work during the Workshop on Partial Differential Equations at IMPA, Rio de Janerio, 1990.

\section{REFERENCES}

[BT1] M. S. Baouendi and F. Treves, A local constancy principle for the solutions of certain overdetermined systems of first-order linear PDE, Adv. Math. Suppl. Stud., vol. 7A in honor of L. Schwartz, Academic Press, Orlando, FL, 1981, pp. 245-262.

[BT2] _ A property of the functions and distributions annihilated by a locally integrable system of complex vector fields, Ann. of Math. (2) 113 (1981), 387-421.

[C] P. Cordaro, Sistemas de Campos Vetoriais complexos, IMPA, Rio de Janeiro, 1986.

$[\mathrm{CH}]$ F. Cardoso and J. Hounie, First order linear PDE's and uniqueness in the Cauchy problem, J. Differential Equations 33 (1979), 239-248.

[H] L. Hörmander, Propagation of singularities and semi-global existence theorems for (pseudo-) differential operators of principal type, Ann. of Math. (2) 108 (1978), 569-609.

[Ho] J. Hounie, Globally hypoelliptic vector fields on compact surfaces, Comm. Partial Differential Equations 7 (1982), 343-370.

[NT] L. Nirenberg and F. Treves, Solvability of a first-order linear differential equation, Comm. Pure Appl. Math. 16 (1963), 331-351.

[RS] J. P. Rosay and E. Stout, Radó's theorem for C-R functions, Proc. Amer. Math. Soc. 106 (1989), 1017-1026. 
[S] H. Sussmann, Orbits of families of vector fields and integrability of distributions, Trans. Amer. Math. Soc. 180 (1973), 171-188.

[T] F. Treves, Approximation and representation of functions and distributions annihilated by a system of complex vector fields, Centre de Mathématiques École Polytechnique, Palaiseau, France, 1981.

Departamento de Matemática, Universidade Federal de Pernambuco, Cidade UniverSitária, 50.740-540 Recife, Pernambuco, Brasil

E-mail address: hounie@dmat.ufpe.br

E-mail address: joaquim@dmat.ufpe.br 